\title{
A perspective on discussing
COVID-19 vaccines:
Efficacy and adverse effects
}

Not a day goes by in clinic without discussions with patients about COVID-19 vaccines. They are glad that they received their vaccinations but want to know if they can safely attend family gatherings over the upcoming holidays. And those who are taking immunosuppressive drugs may have heard that the medications may blunt their response to the vaccine. I emphasize with patients that the vaccines are effective overall in protecting them from becoming ill with COVID and requiring hospitalization. But because I can't guarantee or accurately measure the degree of protection my immunosuppressed patients achieve after vaccination, I emphasize the need to exercise common sense: to mask when in groups indoors, and to consider asking visitors and family members to get a rapid COVID test the day before any gathering.

Not all immunosuppressive medications equally blunt the response to the COVID vaccines. The B cell-directed therapies (eg, rituximab), mycophenolate, JAK inhibitors, and high-dose prednisone pose the greatest concerns, while the anti-TNF medications are not nearly as troublesome. But we cannot count on the available antibody tests to reliably predict the degree of protection. Thus, my recommendations for management of these medications before and after vaccination are for the moment based on immunologic principles and on what I hope is good clinical reasoning.

Completely different discussions take place with patients who are reluctant or completely resistant to receiving COVID vaccines. I try to understand their reasoning, but I point out that our overflowing hospitals are filled with COVID patients, most of whom have not been vaccinated, and that this situation cannot be attributed to chance alone (ie, the vaccines work). A few patients just "don't believe it" and believe that hospitals and "the government" are "making the numbers up." But in most cases, actual dialogue with patients is possible. Of course, the dialogue often lengthens the visit by 5 to 10 minutes, but I feel it has the chance to positively impact the health of the patient and those around them in a lasting way. Time well spent.

I've found particularly engaging the discussions with patients who say, "We don't know enough about the vaccines," or "The vaccines were developed too quickly." It is true that we do not know the 10-year post-vaccination outcomes, nor do we know 10 -year post-COVID outcomes. But as I have thought about this in the clinic and in my role as editor, we actually know a surprising amount or, perhaps more accurately, we have a lot of data. Not all findings and conclusions will turn out to be true, as truth in science is often ephemeral. ${ }^{1}$

Hundreds of millions of COVID vaccines have been administered. Due to the virulence and infectivity of the virus, the efficacy of the vaccines has been relatively quickly demonstrated in both randomized and observational studies. The enormity of vaccine exposure, clinical importance, and the social and political implications have contributed to rapid awareness and study of many possible vaccine adverse events. With virtually all vaccines, side effects tend to occur early after administration. We 
have no reason as yet to think the mRNA vaccines will be different. The literature is already replete with reports of side effects and studies of their pathobiology, even reports of attributed side effects that have occurred with an incidence of only 1 per thousands of vaccine recipients.

In this issue of the Journal, we present a Clinical Picture report of a presumed vaccine reaction ${ }^{2}$ that has been colloquially coined "COVID arm." The authors describe a strikingly inflammatory soft-tissue reaction in the vaccine-injected arm. Although seemingly distinctive, as with all uncommon reactions, it is difficult to be certain whether this reaction is fairly unique to mRNA COVID vaccines, or if it has been highlighted here and elsewhere ${ }^{3}$ because of a hyperacute level of vigilance and heightened desire to share information about these vaccines with the medical community.

As this reaction is infrequent (apparently 1 or 2 per thousand) and self-limited, it should not dissuade people from getting the vaccine. It is also worth noting that various non-vaccine-specific localized reactions can occur after upper-extremity injections. Actual bacterial cellulitis is likely exceedingly rare, particularly with the use of singledose vaccine vials, and it generally manifests 3 to 5 days after the injection. Slightly earlier in timing, more common, and less likely to be spreading is a local firm swelling and ecchymotic reaction to needle trauma. I have seen several patients who suffered subdeltoid bursitis reactions from inadvertent injection of different vaccines into the bursal space rather than into the deltoid muscle. Localized reactions appearing and behaving as (nonbacterial) cellulitis within 1 to 2 days have been reported following zoster, tetanus, and pneumococcal vaccinations. So these subacute reactions cannot be uniquely attributed to the mRNA platform or even to the polyethylene glycol stabilizing component.

The passage of time with the collection and reporting of more data should inform us whether the seemingly rare delayed "hypersensitivity" and other reactions in patients receiving mRNA vaccines are uniquely more common with these vaccines. But for the moment, we should all be vigilant and open-minded about possible adverse reactions to these vaccines and take the time to discuss them with our patients, while emphasizing the demonstrated efficacy of the vaccines.

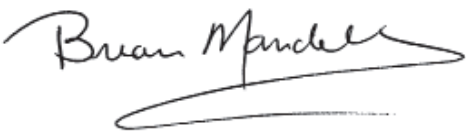

Brian F. Mandell, MD, PhD

Editor in Chief

1. Kuhn TS. The Structure of Scientific Revolutions. Chicago: University of Chicago Press, 1962.

2. Ramalingam S, Arora H, Lewis S, et al. COVID-19 vaccine-induced cellulitis and myositis. Cleve Clin J Med 2021; 88(12):648-650. doi:10.3949/88a.21038

3. Lindgren AL, Austin AH, Welsh KM. COVID arm: delayed hypersensitivity reactions to SARS-CoV-2 vaccines misdiagnosed as cellulitis. J Prim Care Community Health 2021; 12:21501327211024431. doi: $10.1177 / 21501327211024431$ 